\title{
Biosynthetic Convergence of Salinosporamides A and B in the Marine Actinomycete Salinispora tropica
}

\author{
Laura L. Beer and Bradley S. Moore* \\ College of Pharmacy, University of Arizona, Tucson Arizona, 85721 and the Scripps Institution \\ of Oceanography and the Skaggs School of Pharmacy and Pharmaceutical Sciences, University \\ of California at San Diego, La Jolla, CA 92093
}

\section{Supporting Information}

Figure S1. $150 \mathrm{MHz}{ }^{13} \mathrm{C}-\mathrm{NMR}$ spectrum of $\left[1,2-{ }^{13} \mathrm{C}_{2}\right]$ acetate-derived salinosporamide A in pyridine- $d_{5}$ Figure S2. $150 \mathrm{MHz}{ }^{13} \mathrm{C}-\mathrm{NMR}$ spectrum of $\left[\mathrm{U}_{-}{ }^{13} \mathrm{C}_{6}\right]$ glucose-derived salinosporamide A in pyridine- $d_{5}$

Figure S3. Expanded ${ }^{13} \mathrm{C}-\mathrm{NMR}$ signals of individual carbons of $\left[\mathrm{U}_{-}{ }^{13} \mathrm{C}_{6}\right]$ glucose-derived salinosporamide $\mathrm{A}$

Figure S4. $150 \mathrm{MHz}{ }^{13} \mathrm{C}-\mathrm{NMR}$ spectrum of $\left[1-{ }^{13} \mathrm{C}_{1}\right]$ butyrate-derived salinosporamide A in pyridine- $d_{5}$

Figure S5. $150 \mathrm{MHz}{ }^{13} \mathrm{C}-\mathrm{NMR}$ spectrum of $\left[1,7-{ }^{13} \mathrm{C}_{2}\right]$ shikimate-derived salinosporamide A in pyridine- $d_{5}$

Figure S6. $150 \mathrm{MHz}{ }^{13} \mathrm{C}-\mathrm{NMR}$ spectrum of $\left[1,2-{ }^{13} \mathrm{C}_{2}\right]$ acetate-derived salinosporamide B in DMSO- $d_{6}$ 
Figure S1. $150 \mathrm{MHz}{ }^{13} \mathrm{C}-\mathrm{NMR}$ spectrum of $\left[1,2-{ }^{13} \mathrm{C}_{2}\right]$ acetate-derived salinosporamide A in pyridine- $d_{5}$. $\mathrm{X}=$ impurities.

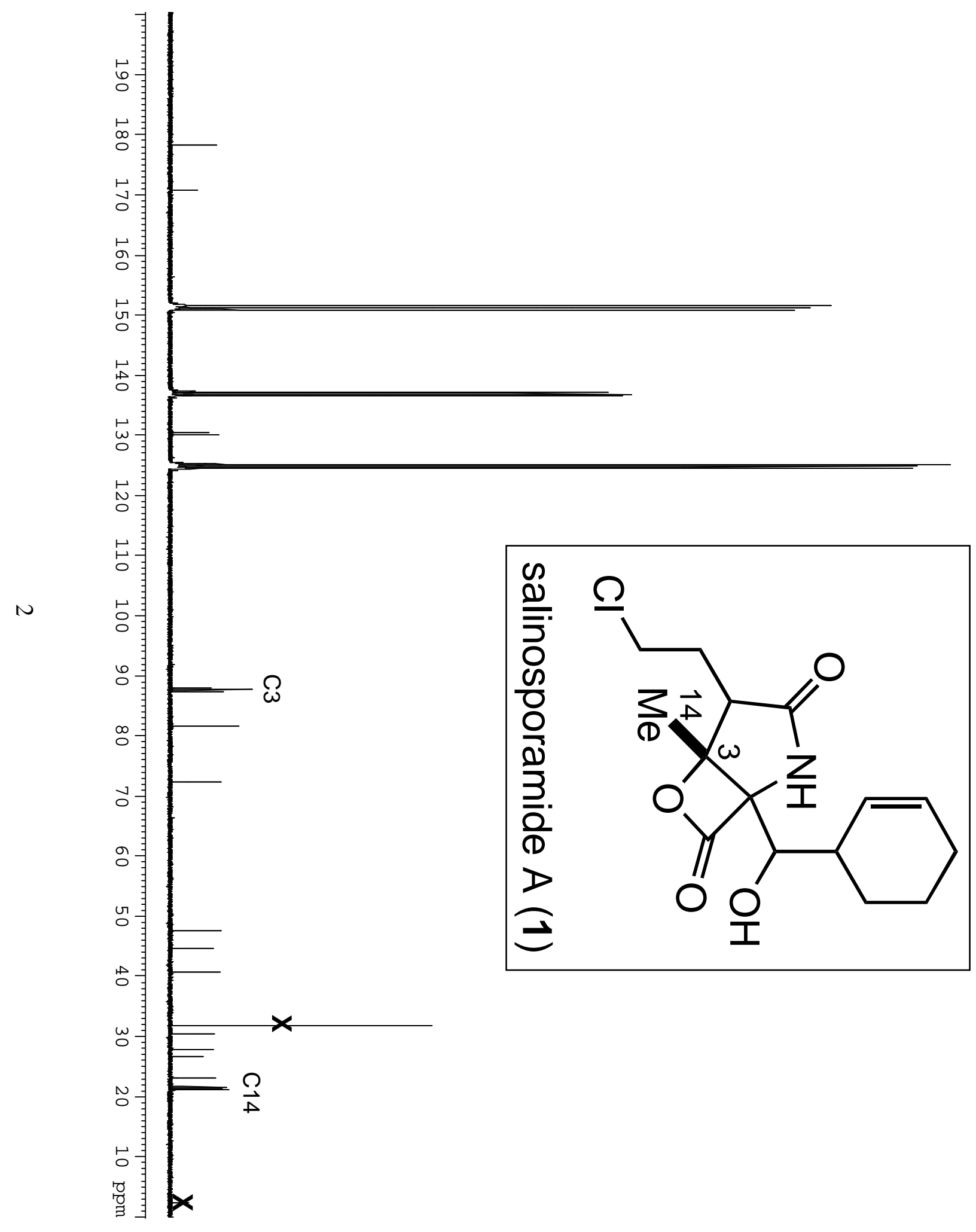




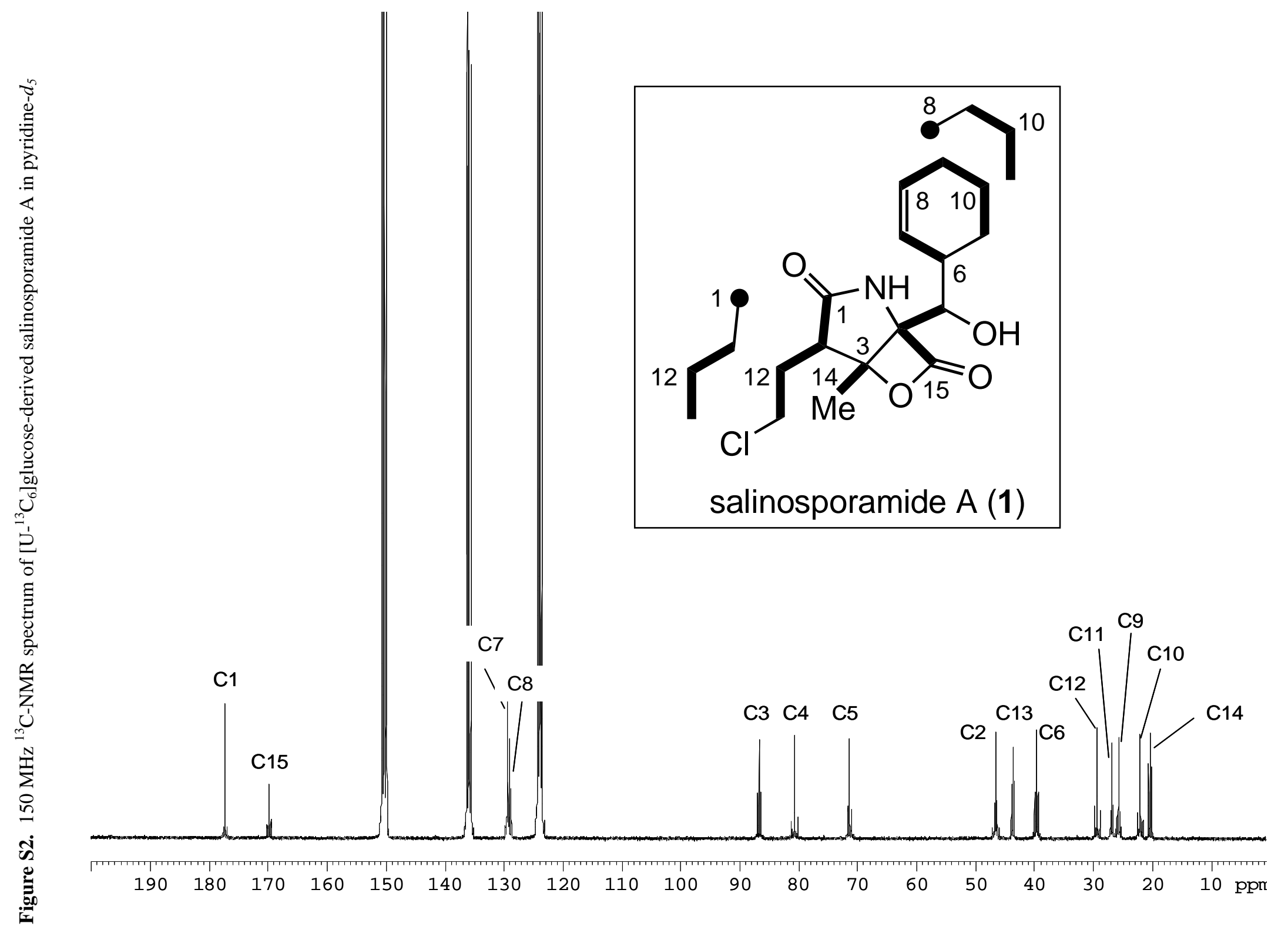



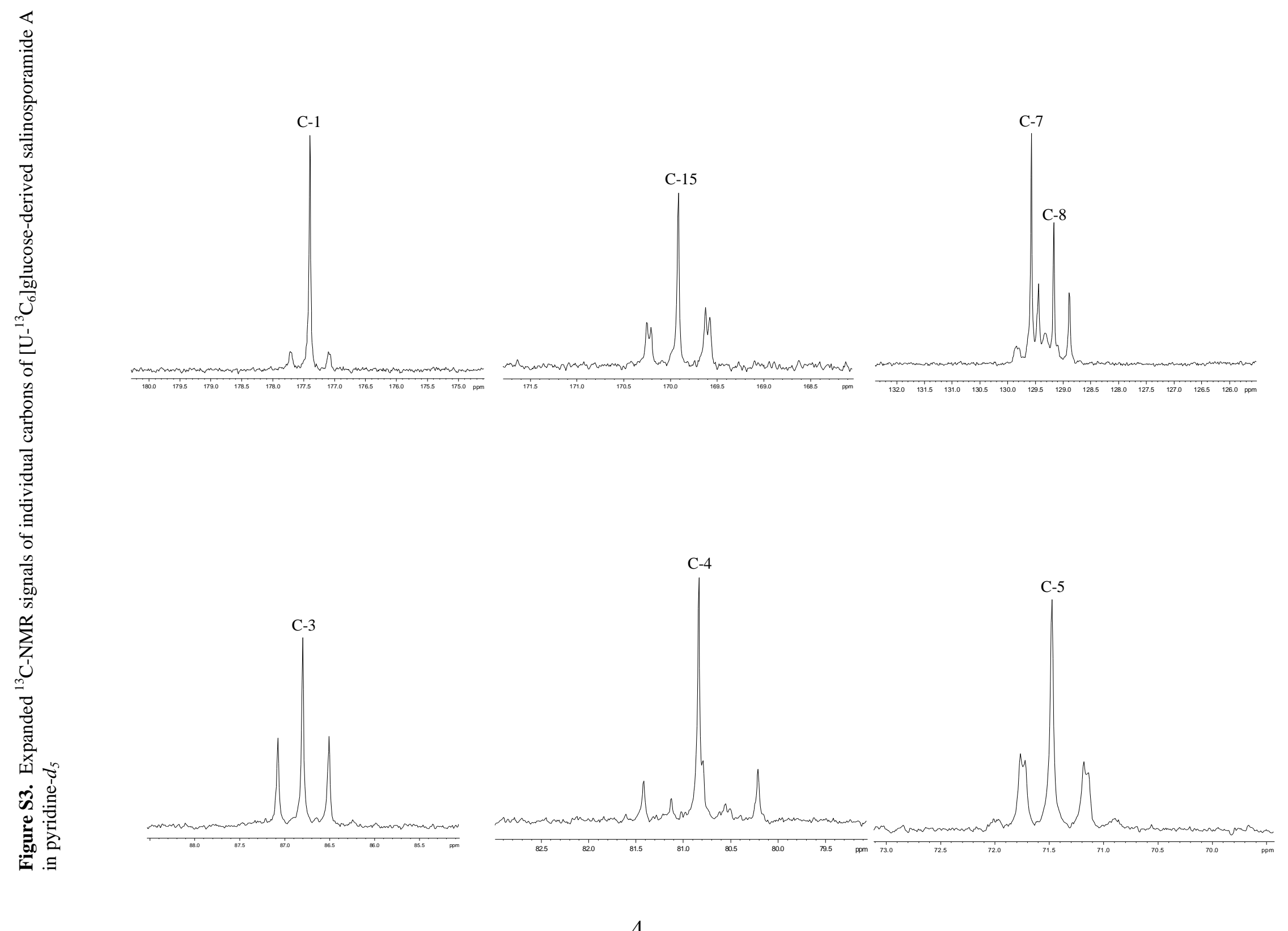

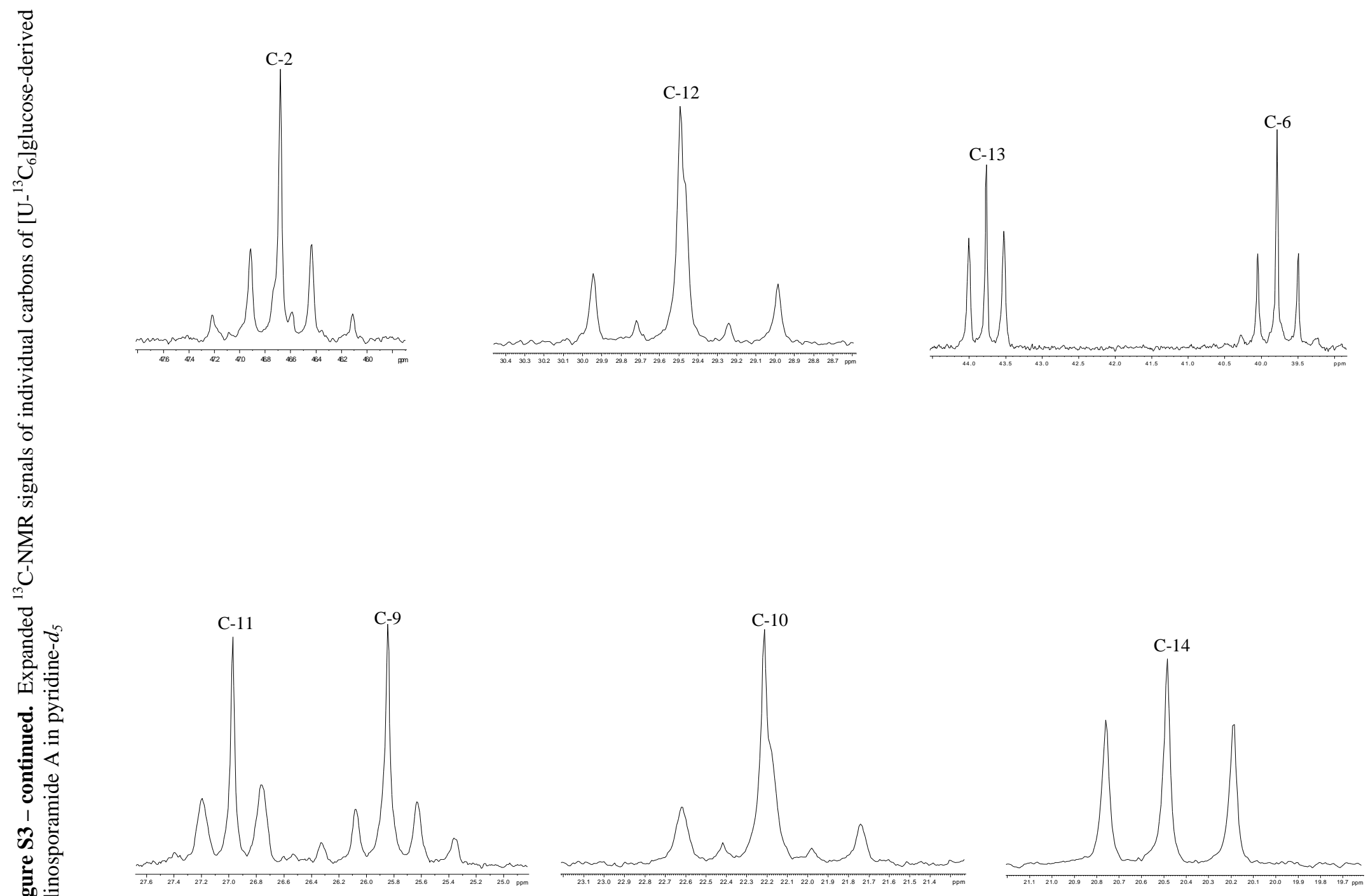

5 
Figure S4. $150 \mathrm{MHz}{ }^{13} \mathrm{C}-\mathrm{NMR}$ spectrum of $\left[1-{ }^{13} \mathrm{C}_{1}\right]$ butyrate-derived salinosporamide $\mathrm{A}$ in pyridine- $d_{5}$

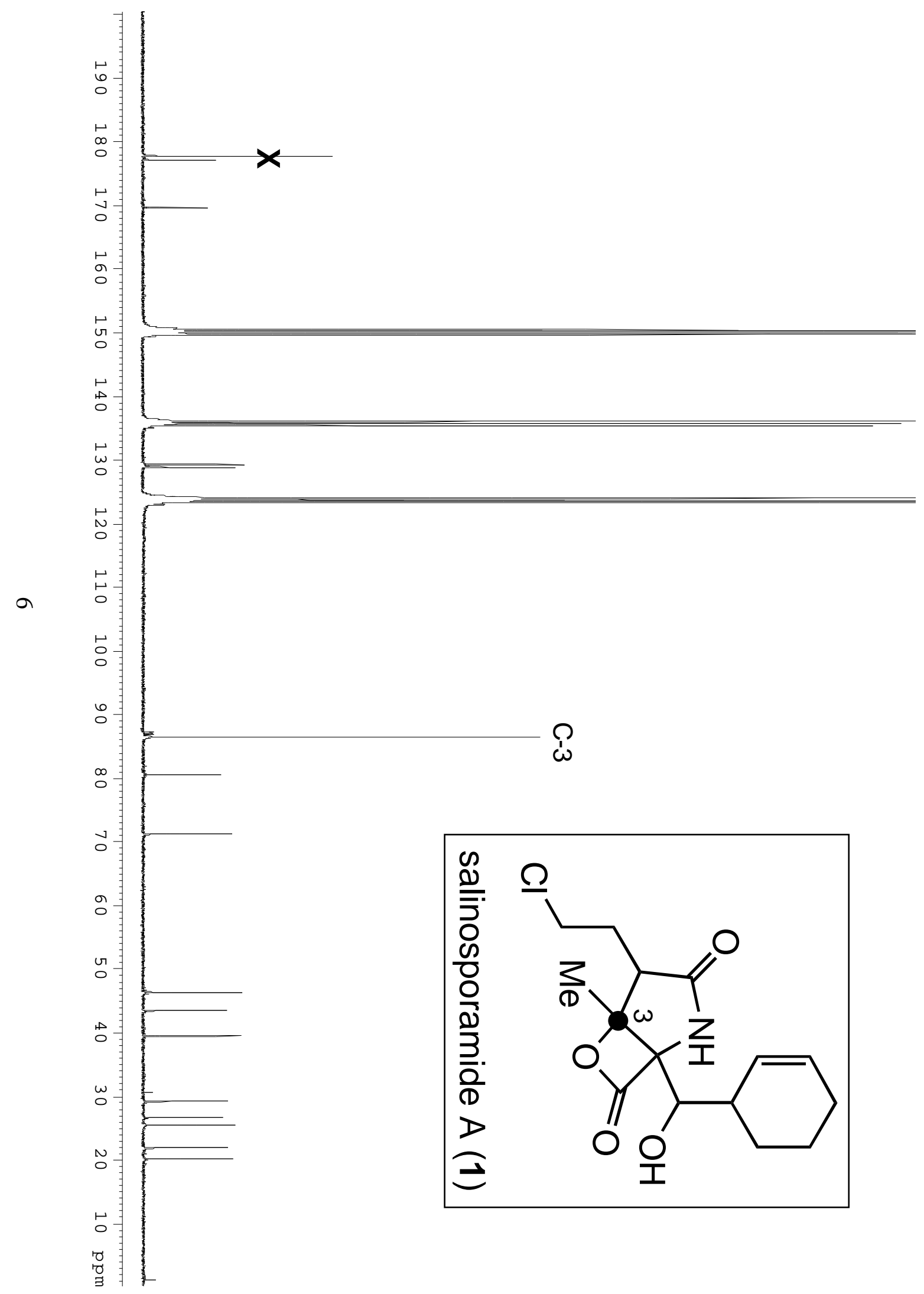


Figure S5. $150 \mathrm{MHz}{ }^{13} \mathrm{C}-\mathrm{NMR}$ spectrum of $\left[1,7-{ }_{-}^{13} \mathrm{C}_{2}\right]$ shikimate-derived salinosporamide $\mathrm{A}$ in pyridine- $d_{5}$

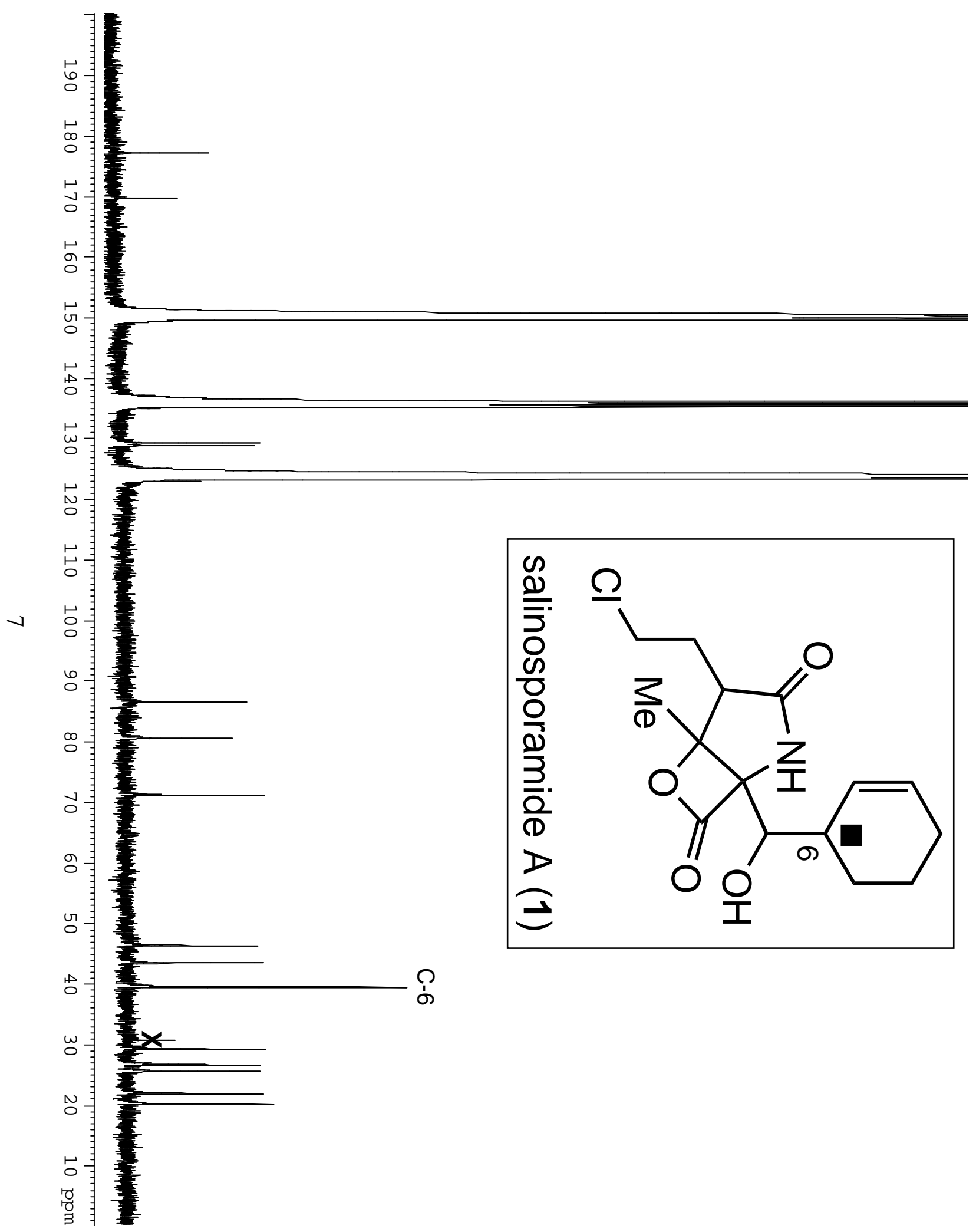


Figure S6. $150 \mathrm{MHz}{ }^{13} \mathrm{C}-\mathrm{NMR}$ spectrum of $\left[1,2-{ }^{13} \mathrm{C}_{2}\right]$ acetate-derived salinosporamide B in DMSO- $d_{6}$.

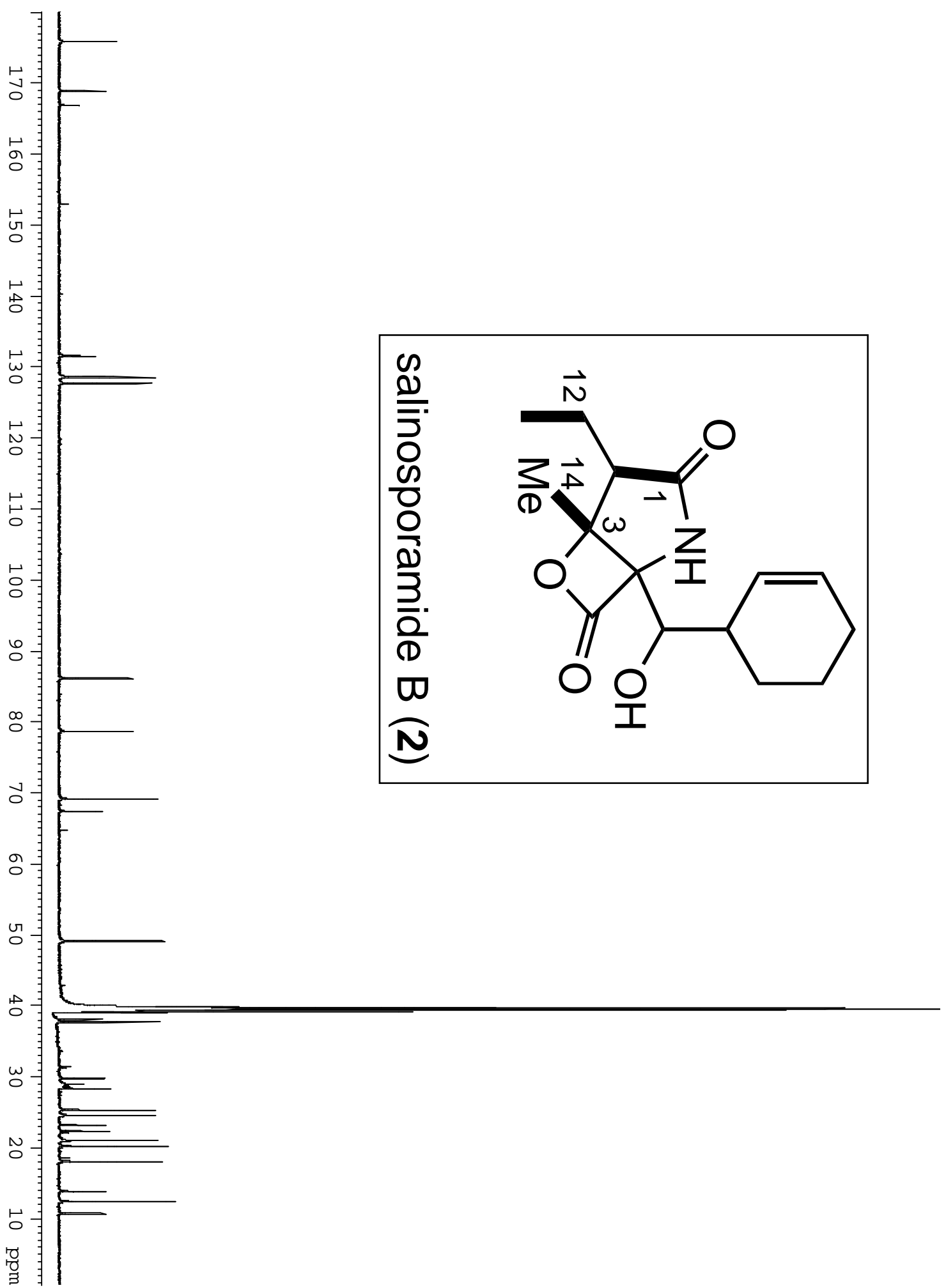

